Einflussmöglichkeiten institutioneller Anleger auf nachhaltige Unternehmensführung

\title{
Mit Pensionen grüne Kasse machen
}

\author{
Pensionskassen entwickeln sich zu einem der wichtigsten Akteure auf den in- \\ ternationalen Aktienmärkten. Ihre Anlagemacht und langfristige Investitions- \\ perspektive können nachhaltige Entwicklung wirkungsvoll voranbringen. Im \\ Folgenden werden die Chancen und Grenzen eines Engagements von Pensions- \\ kassen im Marktsegment nachhaltiger Kapitalanlagen bewertet.
}

\section{$\mathrm{V}$}

Von Ingeborg Schumacher-Hummel erglichen mit der großen Anzahl an Erhebungen zu nachhaltigen Geldanlagen (Socially Responsible Investment, SRI) für Individualanleger gibt es wenige Daten zum institutionellen Markt. Dies liegt daran, dass viele Pensionskassen nicht in Fonds investieren, sondern ihr Vermögen selber verwalten oder in Form von individuellen Mandaten vergeben, deren Größe normalerweise nicht veröffentlicht wird. Sparkes beschreibt das Volumen der Publikums-SRI-Anlagen als Spitze eines Eisberges: "Man kann nur circa 20 Prozent des Gesamten sehen - verglichen mit dem institutionellen Vermögen" (1). In Großbritannien werden laut Sparkes beispielsweise zusätzlich zu den 3,3 Milliarden britische Pfund in Fonds 23,5 Milliarden britische Pfund durch Kirchen und Stiftungen sowie 25 Milliarden britische Pfund durch Pensionskassen nach sozialökologischen Kriterien verwaltet.

\section{- Aktionärsrolle ist zentral}

Die Analyse von aktiven Pensionskassen zeigt in vielen Fällen, dass ihr SRI-Engagement auf einer aktiven, öffentlichen Aktionärs-Rolle basiert oder durch eine direkte Beeinflussung auf die Governance-Strukturen der Unternehmen im Portfolio ergänzt wird. Die treibenden Kräfte sind ähnlich: neben politischen Überlegungen zielt die Verbesserung der Führungs- und Kontrollstrukturen auf eine positive Beeinflussung der finanziellen Performance.

Dem entspricht die Tatsache, dass unter der begrenzten Anzahl von Akteuren vor allem öffentlich-rechtliche Pensionskassen zitiert werden. Bekannte Exponenten sind: die amerikanischen Pensionskassen Calpers (California Public Employees' Retirement System) und TIAA-CREF (Teachers Insurance Annuity Association-College Retirement Equities Fund), die britische Kasse USS (Universities Superannuation Scheme) sowie die CIA (Pensionskasse des Genfer Staatspersonals) in der Schweiz. Diese Vorsorgeeinrichtungen verwalten entweder Pensionsgelder von Universitätsangehörigen oder öffentlichen Angestellten und vertreten damit Berufsgruppen mit einem höheren politischen Interesse.

\section{- Pensionskasse ist idealer Investor}

Warum eignen sich Pensionskassen oder institutionelle Investoren überhaupt für eine aktive Rolle als Aktionäre? Pensionskassen werden mitunter als "ideale Eigner" definiert (2), da ihr Investment durch die Größe und die langfristige Anlageperspektive sehr nachhaltig ausgerichtet ist. Durch ihren quasi-ewigen Anlagehorizont könnte ihr Interesse so weitsichtig sein, die langfristige Perspektive der Unternehmen zu betrachten und damit auch die Interessen von Mitarbeitern, Kunden, Lieferanten und der Gesellschaft einzubeziehen. Für eine aktive Rolle im Bereich SRI bestehen eine Reihe von Anreizen:

- Wie die Argumente für Corporate Governance und SRI aufzeigen, kann Shareholder-Aktivismus die Unternehmensgewinne erhöhen und so die Erträge für beteiligte Investoren verbessern. Während viele Investoren kurzfristige Interessen verfolgen, sind Pensionskassen eher an der langfristigen Wertsteigerung eines Unternehmens interessiert. Durch die langfristige Anlageperspektive können Pensionskassen von den Performance-Vorteilen eines auch auf ökologischethischen Zielen ausgerichteten Engagements profitieren.

- Durch die Größe und Anlagekompetenz sind institutionelle Investoren besser informiert und erreichen durch Skaleneffekte günstigere Kostenstrukturen als kleine Investoren. Die Positionen von Pensionskassen sind groß genug, um die Kosten einer detaillierten Analyse und von entsprechenden Interventionen zu tragen. Bei individu- ellen Investoren besteht eher ein Trittbrettfahrerproblem, das ein aktives Aktionärstum ökonomisch unattraktiv macht.

- Der Trend zur Indexierung macht Pensionskassen zu universellen und permanenten Aktionären: "If you can't sell, you must care." Durch die passive Verwaltung nach einem Index sind Investoren, um das Risiko zu begrenzen, zur Replizierung der wichtigsten Marktpositionen verpflichtet, anstatt eine aktive Titelwahl durchführen zu dürfen.

- Ein wichtiger Anreiz für Shareholder-Aktivismus besteht in gesetzlichen Verpflichtungen wie die Verpflichtung für US-Pensionskassen zur Stimmrechtsausübung (3).

- Initiativen wie die Kampagne "Ethics for USS" stellen ein gutes Beispiel für den Druck seitens der Versicherten eines großen Pensionsfonds dar (4). Sie wurde 1998 aufgelegt, um USS zu einer umfassenden ethischen und ökologischen Anlagepolitik zu bewegen. Die Initiative wurde von 3.500 Mitgliedern sowie von der Association of University Teachers gestartet und scheint erfolgreich zu sein.

\section{Grenzen des Engagements}

Trotz der Argumente für aktives Aktionärsverhalten seitens institutioneller Investoren ist die Anzahl der Gründe für passives Verhalten von Pensionskassen-Managern sehr lang. Die Verpflichtung zur Gewinnmaximierung bringt Bedenken mit sich, dass die Implementierung einer SRI-Anlagestrategie durch positive oder negative Auswahl zu Performance-Einbußen führt, da eine Ausschlussstrategie höhere Transaktionskosten bedeutet und ein Screening mit einer geringeren Diversifikation einhergeht. Die Erfahrungen mit SRI-Fonds und Indizes, die durch ein positives Auswahlverfahren erstellt werden, widerlegen häufig die Befürchtung einer schlechteren Rendite. Gleichzeitig zeigen empirische Analysen häufig ein höheres Risiko der Fonds, das sich durch eine Abweichung in der Länder- und Branchenallokation der Portfolios ergibt - etwa durch ein Untergewicht US-amerikanischer Titel oder die Übergewichtung von Technologieaktien. Kritiker betonen, dass sich nur Pensionskassen mit sehr hohem Deckungsgrad in SRI engagieren können, um dieses höhere Risiko eingehen zu können (5).

\section{Zu wenig Ressourcen für SRI}

Ein Problem gilt auch für große Investoren: Der Zwang zur Diversifikation und die generelle Präferenz für Großunternehmen, um zur Wahrung 
der Liquidität größere Beteiligungen an einzelnen Unternehmen zu vermeiden, reduziert die Motivation einer aktiven Einflussnahme, da sich die Kosten für relativ kleine Positionen kaum auszahlen. Außerdem führen die fehlende Verpflichtung für institutionelle Investoren, zu Themen von besonderem Interesse für Mitarbeiter oder die Gesellschaft Stellung zu beziehen oder praktische Probleme, über ausländische Positionen informiert zu bleiben, zu einer eher defensiven Rolle. Der Forschungsbericht "Just Pensions" fasst die Passivität so zusammen: "Für viele institutionelle Investoren besteht der primäre Hinderungsgrund für SRI nicht in einem Widerstand gegen die Idee, die sich wirtschaftlich noch auszahlen kann, sondern in der offensichtlichen Problematik, sie mit den begrenzten, verfügbaren Ressourcen umzusetzen" (6).

Der gesetzliche Rahmen für Pensionskassen übt einen wichtigen Einfluss auf ihre Größe und Anlagestrategie aus, sowohl hinsichtlich der Anlagenaufteilung wie auch auf die Offenheit hinsichtlich nicht-finanzieller Kriterien wie soziale und ökologische Faktoren. Die Einführung von Deklarationspflichten für Pensionskassen über die Kapitalanlage nach ethischen und ökologischen Kriterien zeigt das wachsende Interesse von Regierungen in Europa und Australien, den Finanzmarkt für eine nachhaltige Entwicklung zu nutzen.

Deklarationspflichten bieten Potenzial, jedoch zeigen Studien große Lücken zwischen Absichtserklärungen und erfolgter Umsetzung. Zwar haben sich beispielsweise in Großbritannien viele Pensionskassen zur Berücksichtigung ökologischer und ethischer Kriterien verpflichtet, gleichzeitig sind nur relativ wenige SRI-Mandate vergeben oder entsprechende Fachpersonen eingestellt worden. Außerdem fehlt beim in Großbritannien relativ beliebten Verfahren des Engagements öffentliche Transparenz. Zwar erfordert der Dialog mit dem Management Diskretion, in vielen Fällen erfolgt jedoch weder ein systematisches Monitoring der Aktivitäten noch eine externe Berichterstattung.

\section{Treiber der Nachhaltigkeit?}

Zurück zu der Frage, ob Pensionskassen zum neuen Treiber in Richtung Nachhaltigkeit werden können. Die Antwort lautet: Ja, aber... Aufgrund ihres Vermögens sind Pensionskassen in den meisten Kapitalmärkten die größte Quelle für langfristige Investments. Durch die Ergänzung staatlicher Rentensysteme um betriebliche und individuelle Vorsorgelösungen wird ihre Größe mittelfristig weiter zunehmen. Die Analyse verschiedener Märkte zeigt ein steigendes Interesse von Pensionskassen am SRI-Segment, das sich allerdings im Vergleich zum Gesamtmarkt noch auf niedrigem Niveau befindet. Die Lücke erklärt sich teilweise durch die Zurückhaltung beim aktiven Engagement als Aktionär hinsichtlich Corporate Governance, aber auch durch finanzielle Überlegungen und fehlendes öffentliches Interesse. Der Gesetzgeber kann, wie in Großbritannien, Pensionskassen zur stärkeren Auseinandersetzung mit ethisch-ökologischen Anlagekriterien oder, wie in der Schweiz, zu einer Aussage bezüglich der Wahrnehmung ihrer Stimmrechte zwingen.

Entscheidend ist das Selbstinteresse der Investoren. Kassen mit stark sensibilisierten und en- gagierten Mitarbeitern sind eher geneigt, ihre Rolle als Aktionär pro-aktiv auszuüben. Daher ist neben der stärkeren Information der Marktteilnehmer eine klare Erfolgsgeschichte von nachhaltigen Kapitalanlagen wichtig, sowohl hinsichtlich des finanziellen Erfolgs als auch des ökologischen und sozialen Nutzens.

\section{Anmerkungen}

(1) Vgl. Sparkes, R.: A business outlook on SRI - Or seeing the wood for the trees. Speech at Triple Bottom Line Investing Conference in Rotterdam, 2. November 2000.

(2) Vgl. Monks, R./ Minow, N.: Corporate Governance. 2nd Edition, Oxford 2001.

(3) Vgl. Brancanto, C.: Institutional Investors and Corporate Governance: best practices for increasing value. Chicago 1997.

(4) Siehe www.ethicsforuss.org.uk

(5) Vgl. Sturm, A./ Badde, M.: Socially Responsible Investment by Pension Funds. A State of the Knowledge Report. Ellipson AG, Basel 2001.

(6) Green, D.: Just Pensions, Socially Responsible Investment and International Development. London 2001, S. 2. Eine weitere Studie gibt es von Friends of the Earth: Top 100 UK pension funds - how ethical are they? Download unter http://foe.co.uk/pubsinfo/briefings/html/ 20010828104434.html

\section{Die Autorin}

Ingeborg Schumacher-Hummel arbeitet an einer Dissertation zur Rolle von Pensionskassen bei Socially Responsible Investments.

Kontakt: Huttenstr. 10, CH-8006 Zürich.

E-Mail: ingeborg.schumacher@gmx.ch

\section{Das Prinzip aller Dinge ist das Wasser, denn Wasser ist alles und
ins Wasser kehrt alles zurück." Thales von Milet (um 625 - um 547 v. Chr.)}

Die in GAIA versammelten Beiträge zeigen, wie Wissenschaft, Wirtschaft und gesellschaftliche Organisationen Probleme der Nutzung, der Verteilung und der Qualität von Wasser analysieren und in Lösungen übersetzen. Namhafte Autoren kommen zu Wort: So betrachtet zum Beispiel Ellen Wiegandt (IUKB, Sion) die nachhaltige Wassernutzung aus globaler Perspektive; Catherine Ferrier (Universität Genf) zeigt, dass durch erfolgreiches Marketing die Konsumenten vermehrt Wasser aus Flaschen statt aus der Leitung trinken; Kristin Schirmer und Co-Autoren (UFZ Leipzig-Halle) widmen sich den Gefährdungen durch Krankheitserreger im Wasser; Donald Tillman (SAM, Zürich) richtet den Blick auf Wasser als Anlageprodukt auf den Finanzmärkten.

Abonnement von vier Ausgaben für Einzelpersonen: 79,00€ (+ Online-Abo 118, $50 €)$,

Institutionen: 139,00€ (+ Online-Abo 208, $50 €$ ), Studenten: 50,00€ (+ Online-Abo $75 €$ ), Einzelheft: $20,00 €$

Erhältlich bei CONSODATA ONE-TO-ONE, Semmelweisstraße 8, D-82152 Planegg, Fon ++49/(0)89/8 57 09-155, Fax -131, kontakt@oekom.de 
(c) 20I0 Authors; licensee IÖW and oekom verlag. This is an article distributed under the terms of the Creative Commons Attribution Non-Commercial No Derivates License (http://creativecommons.org/licenses/by-nc-nd/3.o/), which permits unrestricted use, distribution, and reproduction in any medium, provided the original work is properly cited. 